\title{
El legado olvidado de Fernando Higueras y Antonio Miró: la Casa Rufino. Innovación constructiva y adaptación paisajística
}

\author{
The unrecognized legacy of Fernando Higueras and Antonio Miró: Casa Rufino. \\ Constructive innovation and landscape adaptation
}

Esther Mayoral-Campa $^{(* 2}{ }^{(\text {, Javier Navarro-de-Pablos }}{ }^{(* *)}$

\section{RESUMEN}

A finales de la década de los sesenta, los arquitectos Fernando Higueras (Premio Nacional de Arquitectura 1961) y Antonio Miró elaboran cuatro proyectos en la comarca del Aljarafe sevillano. Este artículo profundiza en el menos conocido y documentado de ellos, la Casa Rufino (Palomares del Río). Para ello, se aporta un material gráfico inédito que ayuda a esclarecer cómo la obra, a pesar de su escasa difusión, asume un papel fundamental dentro de la trayectoria de sus autores. La innovación técnica que supone la utilización de las tabiquillas, el acceso a la casa desde cotas inferiores o la manipulación topográfica se convierten en recursos habituales en obras posteriores. Los hallazgos que se exponen buscan poner en valor un proyecto que, a pesar de su singularidad en el panorama de la arquitectura del siglo xx en Andalucía, muestra un alto grado de vulnerabilidad por su abandono y falta de protección patrimonial.

Palabras clave: Tabiquillas; arquitectura contemporánea; hexadecágono: planta radial; Sevilla; hormigón.

\section{ABSTRACT}

At the end of the 1960s, the architects Fernando Higueras (National Architecture Award of Spain 1961) and Antonio Miró designed four projects in the sevillian region of Aljarafe. This article takes a closer look at the least known and least documented of them, the Casa Rufino (Palomares del Río). To this end, unpublished graphic material is provided which helps to clarify how the work, despite its scarce dissemination, plays a fundamental role in the trajectory of its authors. The technical innovation involved in the use of tabiquillas, the lower-level access to the house and topographical manipulation became common resources in later works. The findings on display seek to enhance the value of a project that, despite its singularity in the panorama of 2oth-century architecture in Andalusia, shows a high degree of vulnerability owing to its abandonment and lack of heritage protection.

Keywords: Tabiquillas; Contemporary Architecture; Hexadecagon: Radial Plan; Seville; Concrete.

$\left(^{*}\right)$ Arquitecta. Profesora contratada doctora. Escuela Técnica Superior de Arquitectura, Sevilla (España).

$\left.{ }^{* *}\right)$ Arquitecto. Profesor contratado predoctoral. Escuela Técnica Superior de Arquitectura, Sevilla (España).

Persona de contacto/Corresponding author: fnavarro@us.es (J. Navarro-de-Pablos).

ORCID: http://orcid.org/oooo-0oo3-2085-7020 (E. Mayoral-Campa); http://orcid.org/oooo-ooo2-1315-3087 (J. Navarro-de-Pablos).

Cómo citar este artículo/Citation: Esther Mayoral-Campa, Javier Navarro-de-Pablos (2021). El legado olvidado de Fernando Higueras y Antonio Miró: la Casa Rufino. Innovación constructiva y adaptación paisajística. Informes de la Construcción, 73(564): e422. https://doi.org/10.3989/ ic. 82591

Copyright: (C) 2021 CSIC. Este es un artículo de acceso abierto distribuido bajo los términos de la licencia de uso y distribución Creative Commons Reconocimiento 4.0 Internacional (CC BY 4.0). 


\section{INTRODUCCIÓN}

Entre mayo de 1969 y agosto de 1970 se proyecta y edifica la Casa Rufino en Palomares del Río (Sevilla), una de las obras más desconocidas de Fernando Higueras y Antonio Miró. Su carácter periférico la ha conducido a un doble estado de abandono y originalidad, sin haberse acometido obras de reforma o rehabilitación desde su construcción. La protección ambiental del corredor verde desde el que se accede a la propiedad ha favorecido también su conservación como atalaya contemplativa, resguardada de la urbanización masiva de la comarca del Aljarafe. Este aislamiento físico ha estado acompañado, a su vez, de un vacío divulgativo.

La Casa Rufino es un ensayo tipológico experimental que, partiendo de un diseño estructural y constructivo radical, alcanza una innovación espacial inédita en su contexto histórico y geográfico. El principal desafío del proyecto consiste en afrontar por primera vez una vivienda de planta hexadecagonal, lo que provocará la búsqueda de innovaciones constructivas como la cubierta sobre tabiquillas, convertida a partir de entonces en una invariante de su arquitectura. Esta doble circunstancia convierte al proyecto aljarafeño en un laboratorio de pruebas a pequeña escala, fundamental para entender el ideario proyectual y constructivo de unos autores que transitan al margen de las modas arquitectónicas de su tiempo. Los resultados de esta investigación, desarrollada a lo largo de treinta meses, se publican en un momento en el que la figura de Higueras vuelve a ponerse de relieve con retrospectivas como la exposición organizada en febrero de 2019 en el Museo ICO, "Desde el origen: Fernando Higueras. 1950-2008".

\subsection{Objetivos}

La singularidad de la Casa Rufino dentro de la arquitectura contemporánea andaluza y la influencia que tendrá en los sucesivos proyectos de sus autores le confieren una serie de valores patrimoniales. A pesar de ello, se encuentra desprovista de figuras de protección, sumándose a esto un estado de creciente abandono y la amenaza de la presión inmobiliaria endémica de la comarca del Aljarafe. El recorrido por los proyectos residenciales coetáneos desvela tanto unos aspectos diferenciales como unas invariantes constructivas y estructurales que sitúan a la Casa Rufino, junto al proyecto análogo de la Casa Caparrós, en un punto clave de las colaboraciones entre Higueras y Miró. Además de cubrir el vacío documental del que adolece, esta publicación pretende situar dos proyectos de pequeñas dimensiones como paradigma de la continuidad creativa y multiplicidad de escalas a las que Higueras responde a lo largo de su carrera.

\subsection{Apunte metodológico}

En el desarrollo de la investigación han sido reunidos y digitalizados planos, fotografías y memorias técnicas conservados del proceso de ideación y construcción de la vivienda, repartidos entre el Servicio Histórico de la Fundación Arquitectura del COAM -sección Fondo Higueras (SHFA-COAM, FH) - y el archivo de Antonio Miró de la Biblioteca de la Escuela Técnica Superior de Arquitectura de Madrid (AAM-ETSAM). También se han realizado entrevistas al promotor y morador de la casa hasta 1980, D. José Rufino Martín, y a sus actuales ocupantes, responsables de su salvaguarda. Finalmente, se ha confeccionado la planimetría del proyecto ejecutado, carente de registro documental - a excepción de una sección general y la planta principal-, sobre la base de mediciones in situ y herramientas de dibujo asistido.

\section{LA CASA RUFINO EN LA OBRA DE FERNANDO HIGUERAS Y ANTONIO MIRÓ}

La posición autónoma de Fernando Higueras con respecto a las corrientes y movimientos arquitectónicos de la segunda mitad del siglo xx lo apartan de las principales líneas de investigación y difusión hasta 1997, cuando una exposición monográfica organizada por el COAM rescata su figura. En el catálogo de la muestra, Alberto Humanes habla de la inexistencia de textos firmados por Higueras y de un vacío documental únicamente cubierto por una monografía de Castro Arines (1972), por la publicación Fernando Higueras. Curriculum Vitae. 1959-1984 - promovida por Lola Botia en 1985 y reeditada dos años más tarde por Xarait- y un texto de Félix Candela que es revisado y ampliado para su inclusión en el catálogo. En las últimas dos décadas se han llevado a cabo importantes revisiones críticas de su obra, entre las que destacan la tesis doctoral de Ascensión García Oviés (2015) y el catálogo de la exposición "Fernando Higueras: desde el origen" (2019). En ambas publicaciones, que consiguen reposicionar al personaje y su obra como versos sueltos del panorama nacional, se hace referencia a una arquitectura compleja y básica a la vez, en la que se conjuga un gran número de referencias y recursos con la búsqueda de formas y espacios primitivos.

Esta complejidad no excluye que todas estén regidas por un estricto orden geométrico y una notable continuidad. A este respecto, Darío Gazapo y Concha Lapayese explican cómo parece que sus proyectos se "prolongan unos en otros, en un discurso continuo [...] donde es imposible distinguir entre el inicio y el final" (1). Esta interpretación es reconocida por el propio arquitecto en una de las escasas entrevistas que concede, en la que reclama la importancia de insistir en la obra anterior para así "procurar hacer el nuevo edificio cada vez menos malo [...], sin dejar nunca de ser el mismo proyecto" (2). De esta forma, sus proyectos deben entenderse como un eslabón dentro de una larga cadena en la que cada encargo es utilizado como una oportunidad de explorar con la estructura, la luz y el espacio, pero siempre cohabitando con experiencias y estrategias previamente ensayadas.

La Casa Rufino se proyecta y construye contemporáneamente a la Casa Caparrós ${ }^{1}$, siguiendo un modelo de planta hexadecagonal aplicado por primera vez en un edificio residencial, pero que ya había sido implantado en otras tipologías: tres años antes, la sede del Instituto del Patrimonio Histórico Español (1965) había supuesto la formalización de años de investigación en torno a los módulos estructurales radiales que, gracias a su carácter fractal, podían extenderse "como si de un organismo vivo se tratase” (1). En las casas Rufino y Caparrós no solo se asume una planta análoga a la del IPHE, sino que se reinterpreta el soporte metálico del tragaluz central a través de un sistema de tabiquillas de ladrillo. Se trata, por lo tanto, de una traducción de una solución de un edificio de gran escala a una vivienda unifamiliar, evidenciando la versatilidad de sus principios arquitectónicos. La luz, señalada por Huma-

\footnotetext{
1 Ambas se desarrollan en la década más productiva de Higueras (1965-1975), coincidente con la incorporación de Antonio Miró como estrecho colaborador. No existen publicaciones específicas sobre la obra de Miró, apareciendo siempre asociado a la figura de Higueras.
} 
nes, Gazapo, Lapayese y Ovies como el factor indispensable de su arquitectura, se desprende de una forma o una solución estructural determinadas (Figura 1): considerada un material irrenunciable, Higueras adapta el material y la forma para conseguir la entrada de luz cenital de la sede del IPHE en espacios domésticos reducidos (Figura 1).

Como todas sus obras, la Casa Rufino se nutre de modelos ejecutados y, a la vez, sirve de inspiración para proyectos futuros. Esa continuidad hace que diez años después, en 1978, se repliquen la planta radial y el uso de tabiquillas tanto en un proyecto de 200 viviendas en Carmona (Sevilla), que no llegarían a construirse, y en 1979 en una de las siete viviendas unifamiliares diseñadas en San Lorenzo de El Escorial (Madrid). Ambos proyectos se encuentran únicamente documentados en el catálogo de obras que publica Xarait en 1987, en el que se enumera una escueta lista de proyectos a los que se adjunta una planta y una imagen (3). El escaso peso de la tipología residencial en la bibliografía publicada contrasta con una prolija difusión de los grandes edificios públicos, particularmente de la citada sede del IPHE. En el caso de la Casa Rufino, a este vacío documental se le añade la desventaja de que la concentración de obras de Higueras en Madrid ha hecho que la mayoría de las investigaciones se hayan centrado en las arquitecturas del área metropolitana de esta ciudad, por lo que su doble carácter periférico-dentro de la península y de la propia Sevilla - ha acrecentado su aislamiento y, consecuentemente, su desaparición en las publicaciones.
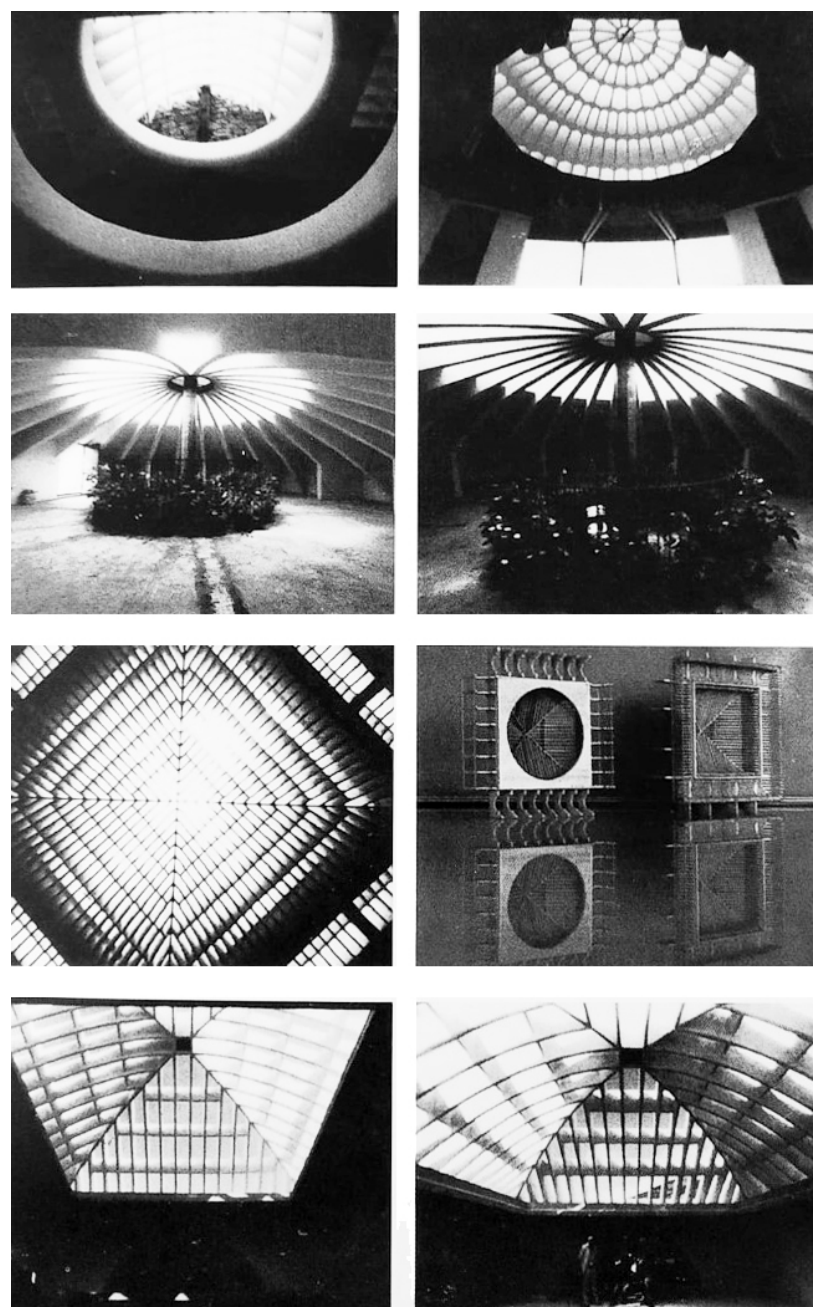

Figura 1. Comparativa de soluciones de cubierta [Fuente: Gazapo de Aguilera, D. y Lapayese Luque, C. (1997). Fernando Higueras, Arquitecturas. Madrid: Fundación Cultural COAM, 45].
Además de en el catálogo de 1987, junto a las viviendas de Carmona, de El Escorial y la Caparrós, la Casa Rufino fue seleccionada por Ubaldo García Torrente y Juan Carlos Reina Fernández como parte de su Guía de arquitectura de Sevilla $y$ área metropolitana siglo $x x$, encargada por el Colegio de Arquitectos de Sevilla en 1992 con el objetivo de catalogar la arquitectura contemporánea de referencia de la provincia. De nuevo, la información gráfica es escasa, aunque se incluye una breve reseña que sintetiza la particularidad tipológica dentro del contexto geográfico a la vez que evidencia el uso de la estructura como matriz proyectual: "Obra de rara tipología dentro del campo de la vivienda unifamiliar. La concepción radial, como base para la apropiación del paisaje rural circundante, se resuelve con una estructura de hormigón visto, de gran plasticidad, presente desde todos los espacios generados" (4).

Las variantes constructivas que introduce, unidas al escaso material publicado, permiten situar la Casa Rufino como una producción más dentro de un continuo proceso de experimentación en el que cada obra supone una innovación; a través de ella, apoyándose en estrategias recurrentes, se marcarán unas pautas reconocibles en obras sucesivas, formando parte indisoluble e indispensable del imaginario de Higueras.

\section{UNA CASA EN LA CORNISA}

La Casa Rufino se sitúa en el término municipal de Palomares del Río, a $12 \mathrm{~km}$ de Sevilla. Su situación en el territorio está marcada por el límite entre la meseta del Aljarafe y la vega del Guadalquivir. En el encuentro de ambas se encuentra la cornisa del Aljarafe, un desnivel que Higueras y Miró utilizan para construir una casa-mirador. La parcela de la Casa Rufino forma parte de los terrenos de la histórica Hacienda de los Teatinos, un complejo cortijero tradicional. Del total de los $25270 \mathrm{~m}^{2}$ de superficie de la parcela, la vivienda proyectada por Higueras y Miró tiene una ocupación de un $2 \%$, aspecto que refuerza su carácter de arquitectura exenta, con una presencia importante de arbolado, áreas de explotación de ganado ovino y zonas de regadío.

\subsection{El Aljarafe. Variantes tipológicas en un territo- rio por construir}

La fructífera relación de los arquitectos con la comarca del Aljarafe había comenzado en 1968 con el diseño de una barriada social en Mairena. Al proyecto de Ciudad Aljarafe, que incluía 1192 viviendas, un complejo sistema de espacios públicos y un colegio, se le añade el encargo de construir un club social para la futura ciudad-jardín de Simón Verde. Los tres proyectos $^{2}$, incluyendo la Casa Rufino, se localizan en un radio de $1,56 \mathrm{~km}$. En esta reducida porción de territorio, Higueras y Miró ponen en práctica un catálogo de variantes tipológicas, la mayoría de ellas ensayadas previamente en Madrid. Mientras que en la barriada de Ciudad Aljarafe se reconocen aspectos esenciales de la Unidad Vecinal de Absorción de Hortaleza (1963), en el Colegio Aljarafe está presente la huella pedagógica, estructural y espacial del Colegio Estudio de Aravaca (1962-1963). Mientras, en el club social queda patente el uso de galerías perimetrales abiertas como estrategia

2 A ellos habría que incluir la redacción del Plan General de Ordenación Urbana de Palomares del Río, fechado en 1969, aunque no llegaría a ser tramitado administrativamente. 
de captación de luz y ventilación, recurso utilizado en todas las viviendas proyectadas en la década de 1960. Por último, en la Casa Rufino se ponen en práctica recursos como son la manipulación de la topografía en bancales o el ya citado planteamiento del espacio doméstico radial, ambos ensayados paralelamente en la Casa Caparrós (1969). La repetición de estrategias proyectuales no les impide, sin embargo, introducir en cada obra una nueva exploración, motivados por el interés por adaptarse a los materiales de construcción del lugar y al clima extremo del sur. Este propósito determina el uso del ladrillo rústico artesanal de taco de Coria del Río en todas las obras del Aljarafe, una referencia explícita a la materialidad de la torre campanario de la Giralda, visitado recurrentemente por Higueras en sus estancias en Sevilla (5).
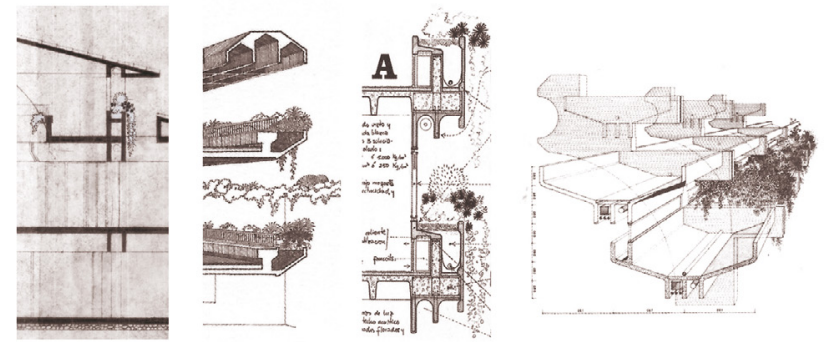

Figura 2. Evolución constructiva de las jardineras: Casa Rufino (1970); Casa María Josefa Huarte (1971); Oficinas en calle Serrano 69 (1979); Oficinas para la firma DOM en Colonia (1980). [Fuentes: AAM-ET-

SAM; Botia, L. y Doval-Sánchez, G. (2019). Fernando Higueras: desde el origen. Madrid: Fundación ICO, Ministerio de Fomento].

El hecho de que el Aljarafe tuviera una explotación del suelo edificado de un $1,78 \%$ en 1970 (6) convertía a la comarca en un territorio por construir. La libertad proyectual con la que los arquitectos afrontan el encargo hace de la Casa Rufino un "laboratorio de experiencias", sin condicionantes urbanísticos ni legislativos. Esta autonomía en el diseño venía acompañada de amplios presupuestos financiados por el mecenazgo de empresarios y constructores como D. José Rufino Martín o D. Jaime García Añoveros ${ }^{3}$, que consideraron la presencia de arquitectos nacionales de prestigio una ocasión para disponer de sus propias viviendas de autor.

A pesar de construirse paralelamente al resto de proyectos, la Casa Rufino es escasamente citada, al margen de la reseña de García Torrente y Reina Fernández. Mientras el Colegio y la Ciudad Aljarafe han sido objeto de publicaciones monográficas (7), la vivienda de la familia Rufino únicamente aparece referenciada en un texto de Juan García-Millán (8), aunque se explica que la casa no llega a construirse. Este error deriva, con probabilidad, de la existencia de un primer proyecto lineal que sería finalmente desestimado por los propietarios.

\subsection{Estrategias de ocupación paisajística. De la es- cala territorial al detalle constructivo}

La intención del matrimonio Rufino era la de disponer de una casa de campo que permitiera a sus hijos estar en contacto con la naturaleza a la vez que desarrollar la actividad ganadera familiar. La construcción del Colegio Aljarafe y el encuentro con Fernando Higueras y Antonio Miró fueron claves para ejecutar el encargo y decantaron, con el visto bueno de los

3 Ministro de Hacienda entre 1979 y 1982, Añoveros era el propietario de la Hacienda de los Teatinos, a 50 m de la Casa Rufino. arquitectos, la elección de una parcela contigua al cortijo de Jaime García Añoveros. Con un camino expedito entre la casa y el colegio, "los niños iban y venían a clase en calesa por el camino de los valles; cruzaban el bosque y volvían sin problemas. El campo era entero para ellos" (9). La integración del proyecto en una cornisa virgen permitió mantener ese carácter rural, alejado de los modelos de periferia característicos de la década (10). El respeto por las preexistencias vegetales, la puesta en valor del entorno y el entendimiento de la silueta topográfica como material de proyecto fundamentan el diálogo con un paisaje todavía virgen: la última de estas estrategias, formalizada mediante un bancal sobre el que se apoya la casa, permite disponer de una visión integral del entorno y, a la vez, resguardarse entre la vegetación ${ }^{4}$.

Además de la transformación del terreno, la presencia de vegetación en los espacios interiores es otro de los recursos recurrentes en la obra de Higueras y Miró puestos en práctica también en la Casa Rufino: no solo los edificios están en contacto directo con los árboles a través de salientes y voladizos, sino que salones y galerías se llenan de pequeños arbustos y macetas. Esta decisión, que aparece por primera vez en los Jameos del Agua de Lanzarote - contando con la colaboración de César Manrique- y se repite en todos los proyectos domésticos posteriores, se ejecuta introduciendo jardineras lineales integradas en los perímetros de las dobles alturas (Figura 2). El resultado son salones, recibidores y galerías en las que se combinan superficies de hormigón en bruto con una nutrida presencia de hiedras y potos colgantes, acompañadas puntualmente de kentias, dracaenas y begonias. Precisamente en las colaboraciones previas entre Higueras y Manrique, ambos habían coincidido en adoptar una estrategia de no intervención del paisaje (11), una posición novedosa y radical en un contexto de expansiones urbanísticas y turismo de masas. Esto explica las invariantes de una implantación integral de sus proyectos, en los que la idea original es reconocible en todas las escalas, desde la territorial a la constructiva.

\subsection{La vivienda como refugio. Procesos comparti- dos de adaptación a la cornisa}

Hasta el momento de recibir el encargo de la familia Rufino, Fernando Higueras había proyectado ocho obras de idéntica tipología: la Casa César Manrique en Camorritos (Madrid, 1962), la Casa Wutrich (no construida, 1962), las casas de Lucio Muñoz y la Arche, en Torrelodones y El Viso (Madrid, 1962-1963), la Casa Santoja, en Somosaguas (Madrid, 1964-1965) - primera vivienda en la que participaron conjuntamente, con Miró consolidado ya como inseparable colaborador (12) - , la Casa Andrés Segovia, en La Herradura (Granada, 1965-1967), la Casa López Villaseñor, de nuevo en Torrelodones (1966-1967), y la vivienda unifamiliar para Nuria Espert en Alcocéber (Castellón, 1968-1971). A excepción

4 El interés por modificar la topografía, presente en la mayoría de sus proyectos residenciales, sería llevado al extremo en su última etapa en las Oficinas Sostenibles en Mallorca (2000) y en la propuesta para los Premios Holcim de arquitectura sostenible en Fuerteventura (2005). Antes, en la Casa Patricio López, en el Puerto de Santa María (1981), el Pabellón de España en Nueva York (1963) o el Rascainfiernos - vivienda y estudio del arquitecto desde su construcción en 1975 hasta su muerte-, ya había demostrado una fijación por estar en contacto con la tierra mediante la incorporación de plantas semienterradas. 
de las Casas Santoja, Arche y Espert, el resto de viviendas se asentaba en terrenos de moderado y fuerte desnivel, lo que propició estrategias de ocupación, transformación y adaptación a la topografía. La solución más extendida consistió en tallar taludes y construir plantas semienterradas sobre las que, posteriormente, se sucedían forjados en voladizo. Esos voladizos y retranqueos, junto a patios y tragaluces, dotaron a estos proyectos de espacios en sombra, estancias exteriores intermedias y de un contacto directo con la vegetación.

Entre 1968 y 1969, durante la construcción de la Casa Espert, Higueras y Miró reciben el encargo de dos viviendas unifamiliares para José Rufino y Rafael Caparrós, proyectos con situaciones de partida muy similares. La parcela de la Casa Caparrós describía un perfil con 9,69 m de desnivel y una pendiente del 12,9\%; análogamente, en la Casa Rufino las diferencias de cota producían un salto de $21 \mathrm{~m}$ y una pendiente del $35 \%$. En ambas posiciones, el paisaje determina el aprovechamiento del terreno: en Palomares del Río, el curso del Guadalquivir y la planicie de las Marismas despejaban una perspectiva privilegiada que, en Torrelodones, quedaba compuesta por el embalse de Los Peñascales y la sierra de Hoyo de Manzanares. Al igual que ocurría en la Casa de Lucio Muñoz, donde la cubierta se fundía con el plano del suelo, en las casas Rufino y Caparrós los elementos naturales y artificiales estaban perfectamente integrados ${ }^{5}$. En ambas se aprovecha el desnivel del terreno para situar una planta semienterrada a modo de garaje con almacén y porche, así como una planta radial poligonal -de doce lados, en el caso de la Caparrós, y dieciséis en la Rufino- que ofrecía un control total del entorno. Esta visión de $360^{\circ}$ que se conseguía al recorrer las galerías exteriores permitía tener la sensación de estar en el bosque en la parte trasera y en un bancal natural en la delantera. A pesar de los intentos previos de construir plantas circulares o concéntricas, no sería hasta la construcción de la Corona de Espinas cuando se ejecutase por primera vez una planta de matriz central.

Se pueden enumerar hasta cinco proyectos no realizados previos a la Casa Rufino en los que Higueras plantea una articulación concéntrica: el Edificio de Restauraciones Artísticas (1961), la Casa Wutrich (1962), el Pabellón de España para Nueva York (1963), el Teatro de la Ópera (1964) y el Palacio de Exposiciones y Congresos (1964). Desde su primer proyecto, la Jaula para elefantes (1954), en el que una "forma helicoidal permite ver los elefantes dentro y fuera de su habitáculo" (13), pasando por las Diez Residencias de Artistas (1960) o el Plan Parcial de Playa Blanca (1963), en los que exploró la repetición infinita del círculo como matriz, su trayectoria queda marcada por el interés de controlar el espacio desde el centro.

A pesar de las muchas similitudes con las viviendas precedentes (Figura 3), que evidencian una estrategia común en la implantación paisajística, existen hechos diferenciales. Así, mientras en todas las viviendas previas se accedía desde las cotas superiores, a la Casa Rufino se llega desde abajo. La combinación de la vegetación preexistente con la plantación de nuevos árboles de gran porte como zapotes, palmeras y cipreses hace que en el primer contacto visual la casa parezca emerger

5 En el expediente del primer proyecto de la Casa Rufino se explicita el interés por respetar la vegetación previa: "Existen en la zona señalada para la construcción de la vivienda varios pinos de gran tamaño, una enorme higuera y dos pozos que se han respetado”. como un elemento natural más ${ }^{6}$. Diez años después, cuando Higueras plantea el proyecto para la vivienda unifamiliar de San Lorenzo de El Escorial -ya sin la colaboración de Antonio Miró, tras su separación en 1975-, consolidará el modelo de inserción paisajística introducido por primera vez en Palomares del Río mediante un acceso a la planta semienterrada de la casa desde las cotas inferiores de la parcela.

\section{UN PROGRAMA, DOS PROYECTOS}

La innovación paisajística de la Casa Rufino se combina con una exploración constructiva en la que Higueras y Miró ponen en práctica por primera vez el sistema de tabiquillas abovedadas colocadas a modo de cimbra para acoger la cubierta radial. Desde que los arquitectos recibieron el encargo a finales de 1968 hasta que finalizaron las obras, dos años más tarde, se sucederían dos versiones distintas del proyecto. Siguiendo un mismo programa - una casa para un matrimonio, sus cuatro hijos y un servicio de dos personas-, fueron capaces de responder a estas necesidades a través de dos modelos opuestos de vivienda.

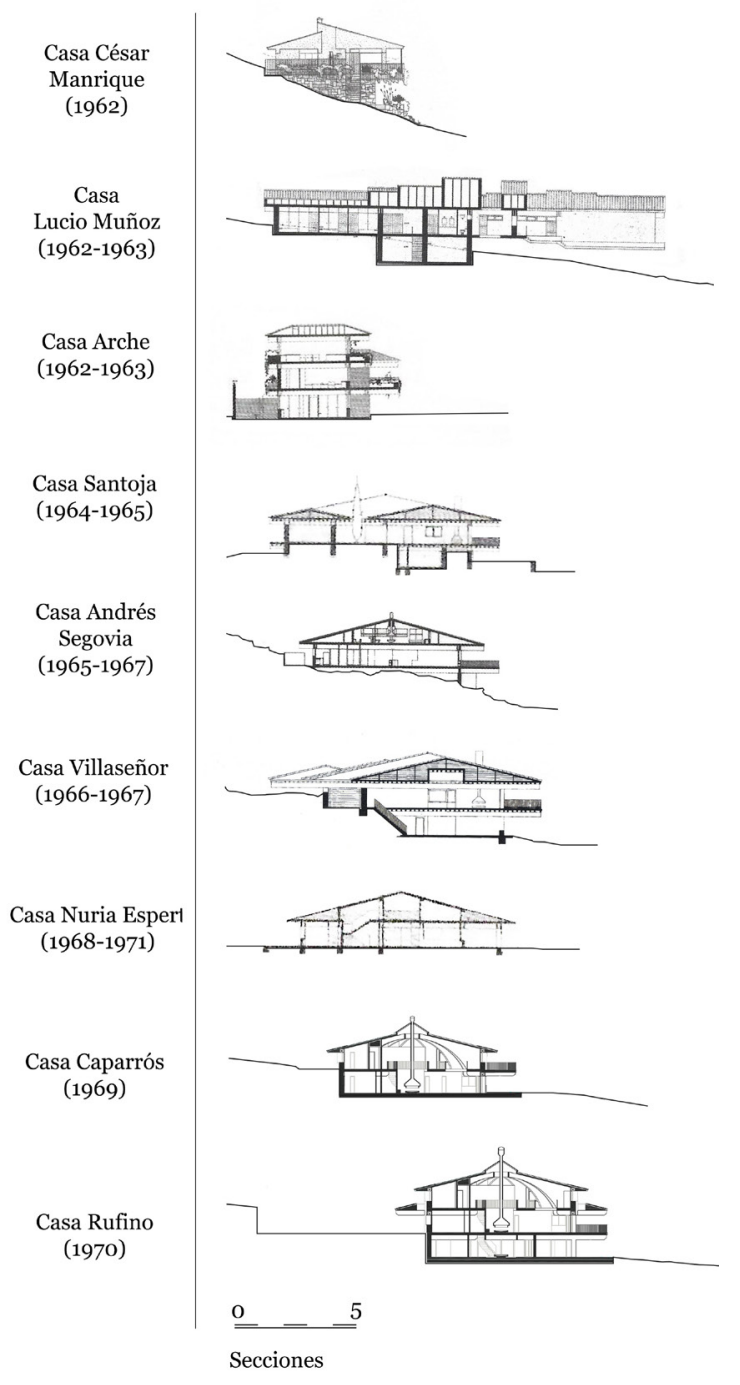

Figura 3. Viviendas unifamiliares desde 1962 a 1969, Fernando Higueras y Antonio Miró. [Fuente: elaboración propia].

6 Esta estrategia guarda similitudes claras con la implantación llevada a cabo dos décadas antes por Lina Bo Bardi en la Casa de Vidrio (1951), de São Paulo, en el que sería su primer proyecto construido. 


\subsection{Primer proyecto (mayo 1969)}

Los primeros bocetos de la Casa Rufino, alojados en el Fondo Fernando Higueras del COAM, muestran un esquema lineal que nunca llegaría a formalizarse. Con un básico definido fechado en mayo de 1969 (Figura 4), el proyecto muestra algunas estrategias que se mantendrían en la segunda propuesta. Entre ellas destacan secciones similares, la construcción de una entalla en el terreno para dar cabida a una planta semienterrada, una estructura de hormigón visto, un cerramiento de fábrica, galerías abiertas y cubierta de tejas árabes a dos aguas. Desde el arranque del proceso hasta la ejecución definitiva, también se evidencia la intención de mirar hacia el valle del Guadalquivir: si bien la primera propuesta se extiende horizontalmente en paralelo al curso del río y la ladera, con un voladizo central dirigido hacia el meandro de Coria del Río, el segundo proyecto mantendrá el voladizo central a modo de mirador. El juego de volúmenes entrantes y salientes permite la entrada de luz en el eje vertebrador de la casa y un ritmo compositivo de la cubierta similar a la de las Casas de Lucio Muñoz, Santoja o Villaseñor.

Con una superficie similar al definitivo, el primer proyecto es presentado en seis planos en los que se detallan los movimientos de tierra, proponiendo la explanación del terreno a una cota de $-1.20 \mathrm{~m}$ sobre la que se asentaría una cimentación de zapatas aisladas y corridas de hormigón armado con cemento de $300 \mathrm{~kg} / \mathrm{m}^{3}$. La integración entre el proceso de ideación arquitectónica y los sistemas constructivo y estructural, presente en toda la producción de Higueras y Miró, se evidencia desde el arranque de la memoria de proyecto: "Toda la forma y organización de la vivienda está condicionada por el sistema constructivo, ya que las vigas resistentes de hormigón tienen forma de V invertida para que sirvan al mismo tiempo que de elementos resistentes, de capialzados de persianas enrollables, dinteles de puertas, ventanas, armarios y elementos de iluminación sobre mesas”(14).

Este fragmento resume la multiplicidad de escalas de un proyecto que, desde la mirada territorial a los detalles cons- tructivos, mantiene una coherencia con un pensamiento arquitectónico que abarca las cinco décadas de producción de Fernando Higueras. A pesar del cambio en la geometría del proyecto definitivamente ejecutado, los principios estructurales, constructivos, espaciales, paisajísticos y medioambientales se mantienen, pasando de un planteamiento lineal a una planta radial.

\subsection{Segundo proyecto (agosto, 1969)}

A pesar del complejo programa de necesidades planteado, los promotores encuentran la primera propuesta demasiado grande y poco compacta. Esto obliga a reformularla hacia un modelo más habitable y de servicios concentrados, con capacidad de satisfacer el deseo del propietario de disponer de un espacio personal segregado que le permitiera aislarse de las rutinas familiares.

Para ello, se decide introducir una planta más en forma de buhardilla, convertida en uno de los puntos singulares de la casa. Este espacio queda fuertemente condicionado por la presencia de las tabiquillas - recurso constructivo que estaba siendo probado coetáneamente en la Casa Caparrós-, que permitían el aprovechamiento de un espacio que se hubiera perdido con una cubierta tradicional de tabiques aligerados. Junto a esta incorporación, el proyecto pasa a articularse mediante una planta radial, lo que favorece el diseño de un espacio concentrado deseado por los propietarios y potencia la idea de la casa como observatorio del paisaje. El segundo proyecto quedaría estructurado, por lo tanto, en tres plantas en forma de hexadecágono, repitiendo el modelo circular de las primeras obras de Higueras. La combinación de la forma y la posición de la arboleda de la cornisa favorecían la idea original de construir una casa emergida de la tierra. La planta semienterrada, la de menor cota, queda asentada en un bancal artificial con el objetivo de extender el espacio interior en los momentos en que las condiciones climáticas fueran favorables (Figura 5). En el interior, la planta queda compuesta por un espacio cerrado central - adosado a la diferencia de cota del terreno- y por un espacio cubierto a modo de porche perimetral resultante de las galerías en voladizo de la primera planta.

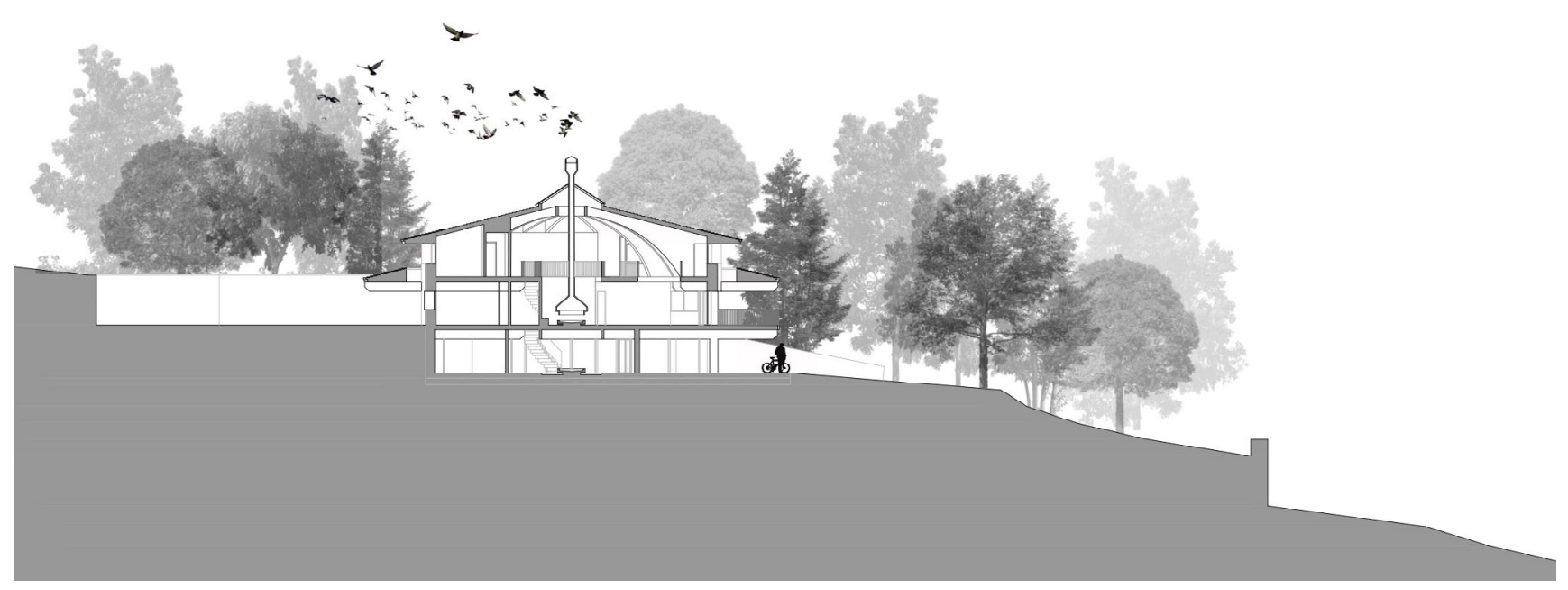

Figura 5. Sección longitudinal del proyecto definitivo [Fuente: elaboración propia]. 

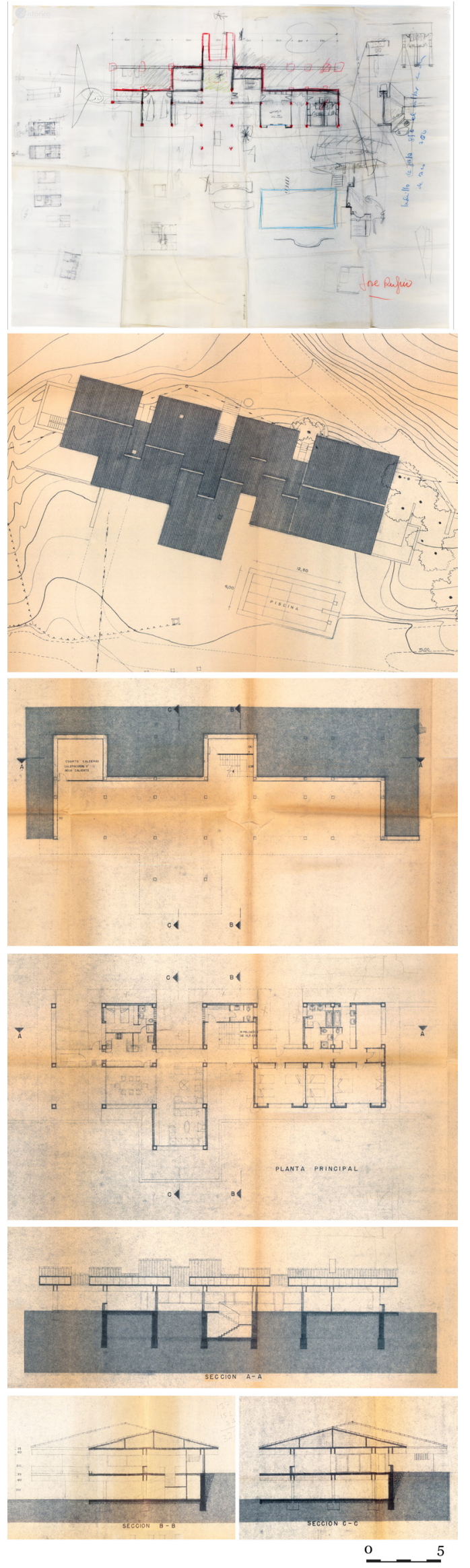

Figura 4. Boceto (superior) y planimetría del primer proyecto de la Casa Rufino. Fernando Higueras y Antonio Miró, 1969. [Fuente: SHFA-COAM, FH/Po27/C010-2-07, 08].
El bancal explanado, abierto hacia la vega del Guadalquivir, es utilizado como garaje abierto en el exterior y también como lugar de esparcimiento en el interior. El espacio central es una habitación de carácter polivalente, que será empleado por la familia y los posteriores moradores como lugar de celebración. En el centro aparece una fuente de hormigón que parece surgir del terreno, en consonancia con el recurrente interés por estar en contacto con la tierra y los recursos naturales. Desde esta sala se accede a la planta superior con una escalera que refuerza el juego geométrico y que significará el principal cambio con respecto a la obra ejecutada: si en el proyecto aparecía al fondo del eje de la casa, asumida por el anillo de estancias, en obra pasa a integrarse en el pasillo en torno a la doble altura central, reforzando el diseño concéntrico de la vivienda.

En la primera planta se concentra un extenso programa familiar. Su articulación sigue la misma geometría de la inferior, dividida en tres anillos concéntricos con diferentes usos y cualificaciones espaciales. En esta planta principal se evidencia la idea de un único proyecto pensado desde todas las escalas: en una secuencia interior-exterior, como si se tratase de los anillos de crecimiento de un árbol (Figura 6), el espacio se dilata desde el núcleo central a doble altura, rematado por un tragaluz, hasta el entorno perimetral en forma de galerías. Entre estos dos anillos radiales se concentra el programa doméstico, con una secuencia de cuatro dormitorios, tres baños, cocina, salón y estudio.

Desde el planteamiento ideológico de Higueras, podría pensarse en el paisaje como el último de esos anillos, introducido en el interior de la casa gracias al control visual de los $360^{\circ}$ circundantes. El espacio central que constituye el primer anillo representa el hogar, donde una chimenea metálica situada en su centro geométrico y rodeada por los espacios más públicos de la casa -el salón y el comedor- refuerza la idea de centro como origen del proyecto. La posición de estas estancias también es intencionada, ofreciendo unas vistas abiertas hacia la vega del Guadalquivir. El recurso de la chimenea como centro de la vivienda será recurrente en la arquitectura doméstica de Higueras y Miró, con el precedente de la vivienda germinal de todas las posteriores, la Casa de

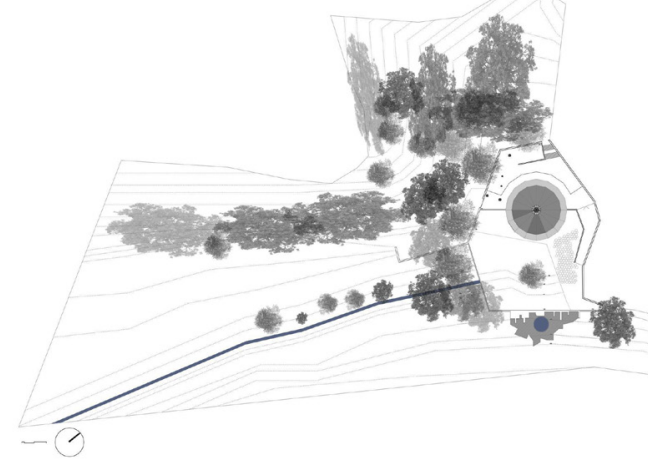

Figura 6. Situación del proyecto definitivo en la parcela [Fuente: elaboración propia].

César Manrique en Camorritos. Al igual que en la posición de la casa con respecto a la llegada desde cotas inferiores se reconocía una referencia a la Casa de Vidrio de Lina Bo Bardi, la posición central de la chimenea - convertida en fuente en la planta semienterrada- es una referencia a la arquitectu- 

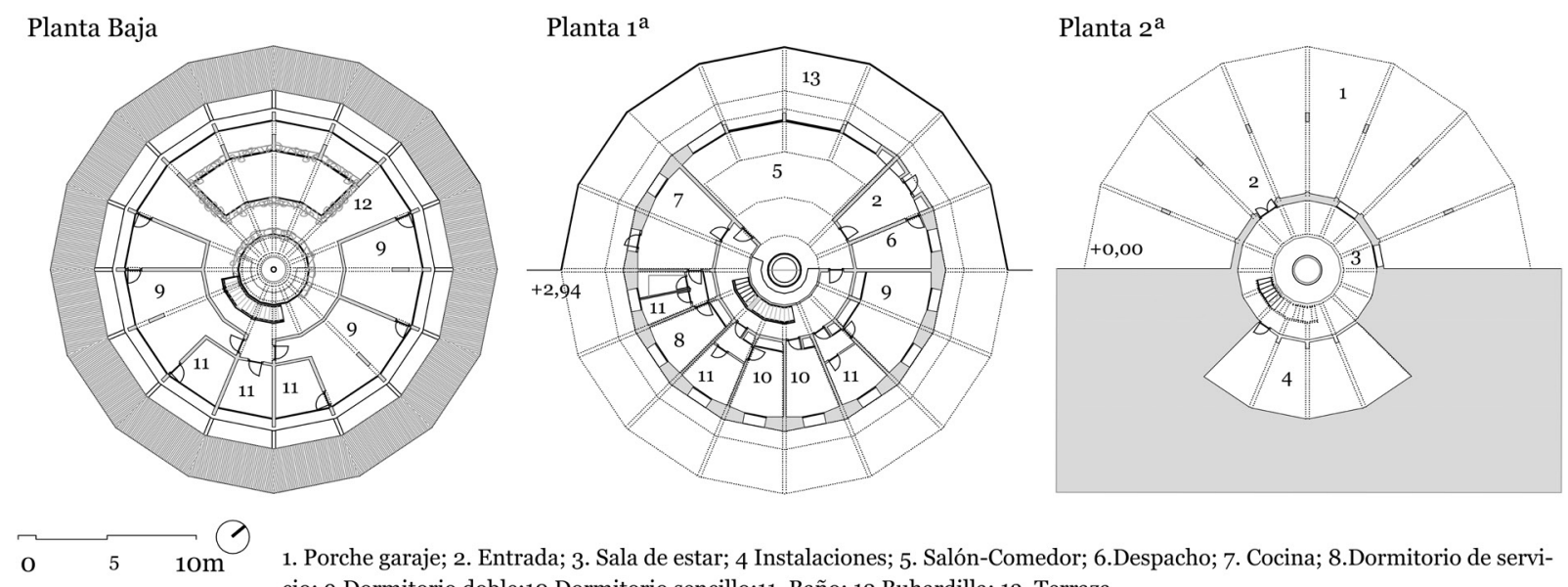

1. Porche garaje; 2. Entrada; 3. Sala de estar; 4 Instalaciones; 5 . Salón-Comedor; 6.Despacho; 7. Cocina; 8.Dormitorio de servicio; 9.Dormitorio doble;10 Dormitorio sencillo;11. Baño; 12 Buhardilla; 13. Terraza.

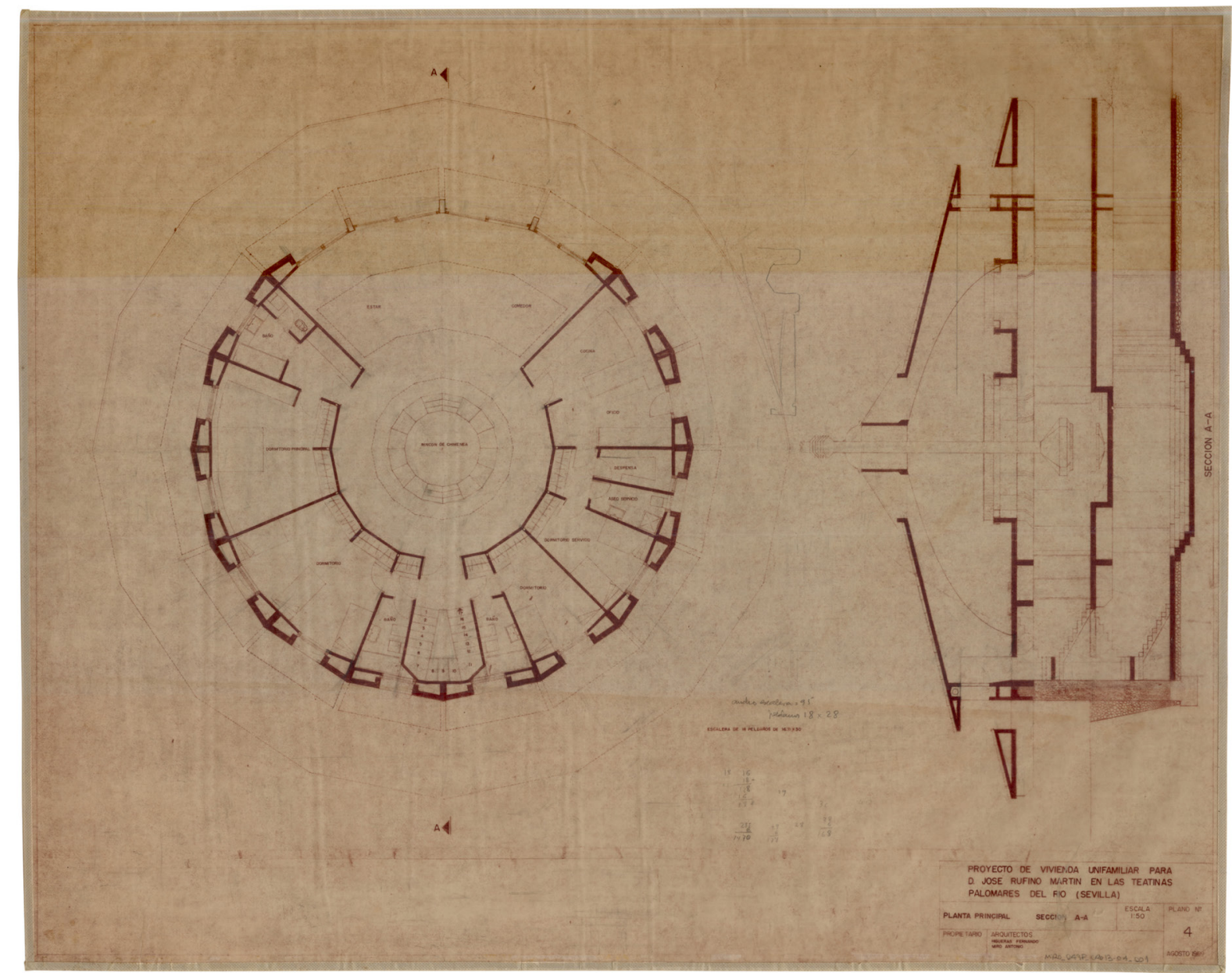

Figura 7. Plantas baja, principal y segunda [Elaboración propia]. Abajo, planta principal y sección del segundo proyecto. Fernando Higueras y Antonio Miró, 1969. [Fuente: AAM-ETSAM, Ref. Casa Rufino, consultado: 20/05/19].

ra doméstica de Frank Lloyd Wright, autor que, junto a Pier Luigi Nervi y Félix Candela, conformaba el trío de referencias ineludibles de Higueras (15). En las Casas Robie (1910) y Wingspread (1939), ambas pertenecientes a la serie de "casas en la pradera", Wright confiere un particular simbolismo al núcleo central de la casa al colocar el fuego - la chimeneaen su centro geométrico. Tanto para Wright como para su “discípulo" español, el fuego actúa como símbolo del refugio primigenio en torno al cual las familias se reúnen.
Prosiguiendo con una lectura radial de la casa, el segundo anillo en la planta principal está ocupado en su zona oeste por el resto del programa (Figura 7): los dormitorios, tanto familiares como los de servicio, la cocina, la entrada a la vivienda y los espacios de conexión, pasillos y escaleras. Todas las estancias se acomodan a la geometría de la planta, lo que resulta en una secuencia de trapecios isósceles que converge en el salón central. El tercer y último anillo consiste en un espacio exterior cobijado bajo la cubierta a modo de galerías. Este espacio 
intermedio, característico de las arquitecturas de Higueras y Miró, amortigua y cualifica las relaciones entre el exterior y el interior de la vivienda. En los proyectos de la Ciudad y el Colegio Aljarafe, la galería perimetral abierta también supuso un elemento indispensable para la articulación de los recorridos y la relación con el paisaje. En el caso del centro escolar, los espacios comunes tenían forma de pasillo abierto en plantas superiores y de plano de sombra en la inferior y, análogamente, la red de espacios públicos de Ciudad Aljarafe estaba compuesta de pasarelas y núcleos abiertos.

La segunda planta de la Casa Rufino está conectada con la primera por la misma escalera semicircular que arrancaba de la planta semienterrada. En su parte central, el forjado se interrumpe creando una doble altura - coincidente con el salón-comedor de la planta principal-, que baña de luz indirecta todas las estancias comunes de la vivienda. En el segundo anillo se sitúan dos dormitorios principales con sendos baños y dos estancias de estudio. Este nivel toma el aspecto de buhardilla, asumiendo la inclinación de la cubierta, sustentada por 16 costillas estructurales de fábrica. Esta solución, además del citado aprovechamiento del espacio que suponía y de la mayor ligereza que procuraba a la cubierta, se adaptaba a la necesidad de una entrada de luz que superase la cota de los árboles de mayor altura. Lejos de ser un mero recurso constructivo, las tabiquillas supondrían un antes y un después en la cualificación espacial de la arquitectura doméstica de Higueras y Miró: la intención de replicar la entrada de luz cenital ensayada en el patio central de la Corona de Espinas motiva la adaptación de una estructura metálica en una de fábrica de ladrillos.

El adoptar tanto en tipologías residenciales como en grandes edificios públicos espacialidades análogas supone una dignificación de la arquitectura menor, poniendo de relieve cómo el proceso de ideación de Higueras huye de lenguajes, estilos y gestos para enfocar sus esfuerzos en la experiencia espacial y sensorial. La Casa Rufino confirma, así, el juego de escalas y formas que se replican, adaptan, crecen y se contraen a partir de un módulo estructural autónomo y una solución constructiva versátil. Ambas componentes acaban por construir unas arquitecturas que, como reconoce Higueras, "son portadoras de dinamismos o energías con poder de expansión, con poder de generación de otras formas y espacios infinitos” (2).

\section{INVARIANTES CONSTRUCTIVAS Y ESTRUC- TURALES: PROYECTAR DESDE LA SECCIÓN}

Entre los años 1969 y 1970, los arquitectos ejecutan un total de siete proyectos. La variabilidad de situaciones, tipologías y escalas les obliga a desarrollar un sistema de trabajo e ideación que consiste en una repetición de un sistema estructural básico y versátil, capaz de adaptarse a distintas geometrías. $\mathrm{Al}$ comparar la sección de la Casa Rufino con otros proyectos se encuentran similitudes que confirman esa apuesta por una "arquitectura ósea", en la que la estructura vertebra y ordena todas las partes de un sistema (Figura 8):

"Así, la estructura debe ser simple, elástica y plenamente integrada, sin supeditarse a una planta que refleje un programa excesivamente definido [...]. El edificio se ha concebido en base a un único material como elemento fundamental de construcción, el hormigón armado. La estructura está pensada de forma que dé, al mismo tiempo, el carácter arquitectónico exterior e interior del edificio. Ha sido diseñada
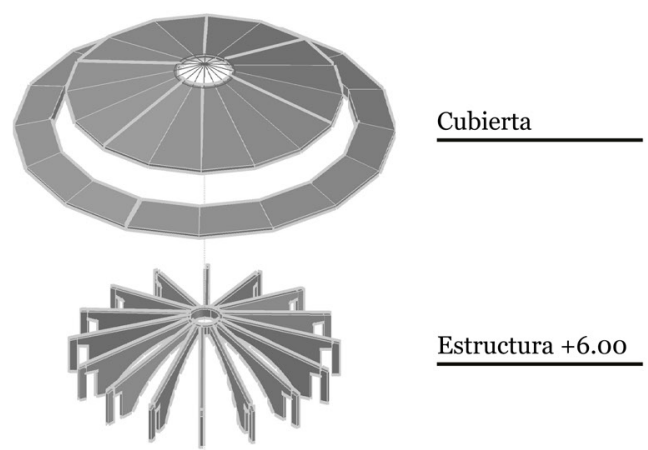

Estructura +6.00

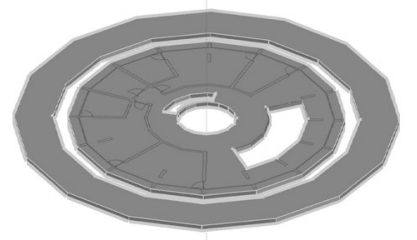

Planta $2^{\mathrm{a}}$

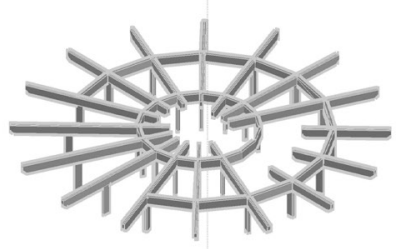

Estructura +3.00

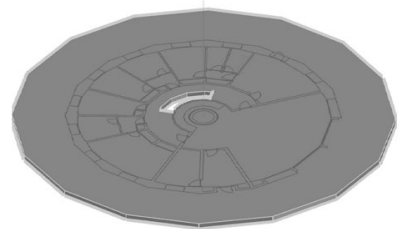

Planta $1^{\mathrm{a}}$

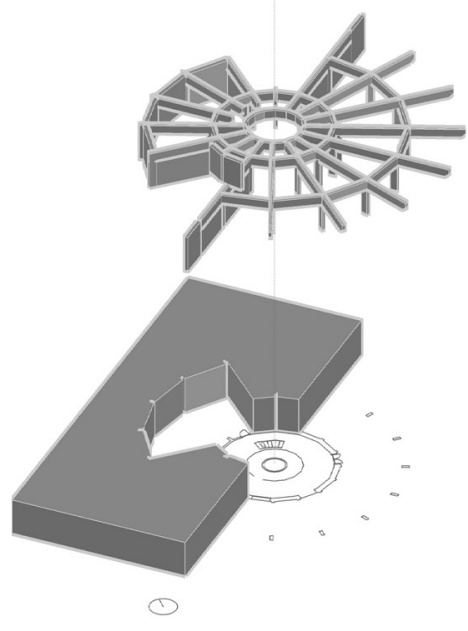

Estructura +0.00

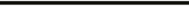

Planta Baja

\section{Figura 8. Axonometría del sistema estructural [Fuente: Elaboración propia].}

cuidadosamente de acuerdo con experiencias anteriores; las vigas tienen el módulo adecuado y son líneas de separación de los espacios. Los arrostramientos transversales forman al mismo tiempo las cajoneras de las persianas y artesonados en crucería en las zonas de pasillos. Todo ello estudiado con una modulación que permite la repetición de encofrados en pilares, vigas y arrostramientos en planta de pisos, manteniendo constante sus secciones" (16).

La cita es aplicable a la mayoría de sus proyectos, incluyendo la Casa Rufino. En este caso, el axioma estructural se traduce 

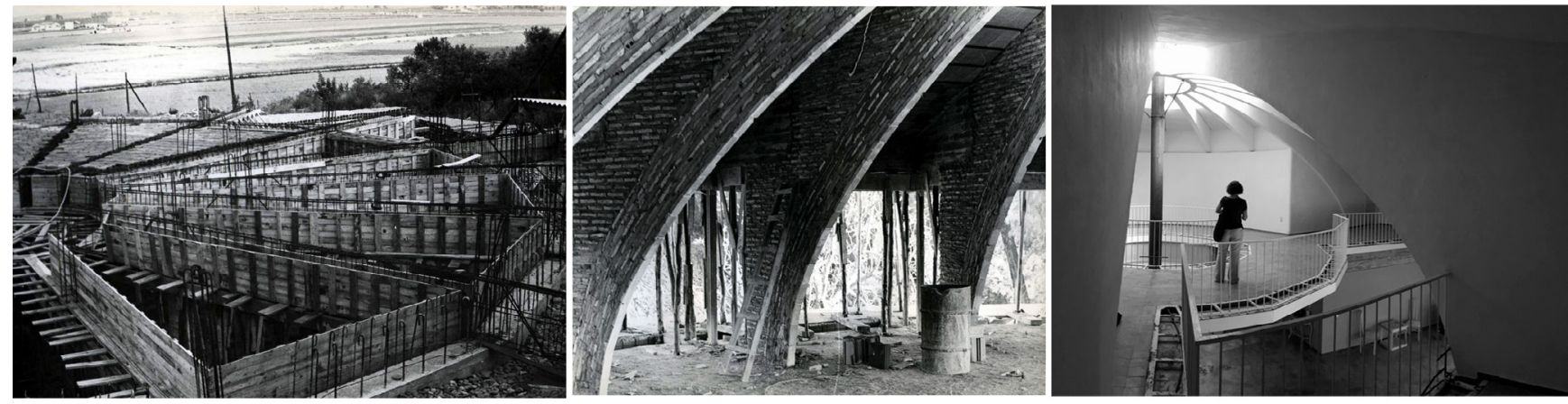

Figura 9: Construcción del forjado principal, tabiquillas y estado actual [Fuentes: AAM-ETSAM, Ref. Casa Rufino, consultado: 20/05/19; Elaboración propia].

en 16 pórticos de hormigón separados a eje $22,5^{\circ}$, compuestos por vigas de sección rectangular con un berenjeno central que da una imagen de doble pórtico, a la vez que aligera la sensación pesada de una sección de $70 \times 20 \mathrm{~cm}$. Biapoyadas, la mayor parte de ellas cuentan con los voladizos que soportan las galerías abiertas.

Con respecto al primer proyecto, se mantiene la idea de que la forma y organización de la vivienda quede condicionada por el sistema constructivo: mientras las vigas de descuelgue sirven para cualificar el espacio - ajustando la altura libre a una escala más doméstica-, dobles vigas de hormigón son introducidas para albergar las persianas, a la vez que se elimina toda transición entre elementos estructurales, constructivos y carpinterías (17) -los pilares están embebidos en los escasos paños de fábrica de forma que los huecos son siempre completos, enmarcados entre elementos estructurales-, la ausencia de mochetas optimiza la percepción del espacio y mejora el flujo entre estancias. Todos estos recursos están en consonancia con dos conceptos fundamentales a la hora de plantear la estructura: en primer lugar, su utilización radical, carente de añadidos o disimulos, con un acabado que se limita al encofrado del hormigón y, en segundo término, el desarrollo de un sistema de elementos apilados que parecen sustentarse gracias a un equilibrio inestable.

A pesar de tratarse de una estructura robusta, con grandes secciones de hormigón armado en su mayoría sobredimensionadas, la sensación al recorrer la casa es la de encontrarse en un espacio ligero y abierto. Este efecto se consigue gracias al citado tratamiento de la luz: con la introducción de las costillas de fábrica o tabiquillas, la luz cenital estará presente en toda la casa combinada con los grandes huecos presentes a lo largo de todo el perímetro radial.

La obra de la Casa Rufino queda documentada fotográficamente con visitas que arrancan el 10 de marzo de 1970, prosiguen el 10 y 24 de abril, el 12 y 28 de mayo, y finalizan con un registro semanal durante el mes de julio. Este intenso seguimiento de la obra - en total, nueve visitas registradas-, considerando el elevado número de encargos que reciben Higueras y Miró ese año, explica la complejidad de la propuesta estructural -que acabaría duplicando el presupuesto inicial-y la implicación personal de ambos en el proyecto. Las investigaciones de García Ovies consideran la utilización de las tabiquillas como uno de los nueve puntos básicos de la arquitectura de Higueras, directamente relacionadas con el interés por "domesticar" la luz cenital:
"En 1966 desarrollan el proyecto para Laboratorio para Cementos Asland [...]. La introducción de la luz cenital en el espacio central de los laboratorios se realiza a través de enormes vigas con sección en ' $Y$ ' conformando una retícula espacial. Esta idea evolucionará incorporando las tabiquillas para la introducción de la luz cenital, de enorme belleza y mucho más económica” (18).

En ese entramado armónico de elementos estructurales y constructivos, en el punto por el que la luz accede al interior y se cruzan con el tiro de la chimenea, aparece una corona de la que parten las costillas y sobre esta un lucernario en forma de paraguas, de mayor pendiente que los paños de las cubiertas de teja. Con la inclusión de esta buhardilla, la casa sirve de paradigma de la arquitectura de Higueras y Miró, pudiendo reconocer siete de las nueve líneas de investigación que propone García Ovies: se trata de una "vivienda unifamiliar", de "estructura radial porticada" girando en torno a un "espacio central" y en la que la "luz cenital" y el "paisaje" se introducen en su interior "mediante una cubierta tipo paraguas y tabiquillas" (Figura 9). Esta secuencia de espacios, detalles constructivos y referencias arquitectónicas clarifica el carácter paradigmático e inédito de la Casa Rufino: en la periferia geográfica y formal, es un proyecto capaz de condensar todas las invariantes de su arquitectura y reinterpretar, a su vez, las singularidades del entorno.

\section{EL LEGADO OLVIDADO Y SINGULAR DE HI- GUERAS Y MIRÓ EN SEVILLA}

El vacío documental en torno a la Casa Rufino no se corresponde con el interés que suscitan sus contrastadas singularidades. Como se ha explicado a lo largo del texto, la introducción de las tabiquillas como solución constructiva parte de una necesidad puramente programática que los arquitectos, con libertad presupuestaria, convierten en una oportunidad para experimentar con los límites del espacio doméstico y el tratamiento de la luz. A pesar de que a partir de las casas Rufino y Caparrós los encargos tienden hacia grandes edificios públicos y concursos, decreciendo el número viviendas unifamiliares, estas adoptarán modelos que parten de los proyectos del Aljarafe y Los Peñascales: tanto las irrealizadas 200 viviendas en Carmona como la de San Lorenzo de El Escorial adoptan una planta radial, se recurre al uso de tabiquillas - en el primer caso- $\mathrm{y}$ al acceso inferior de la parcela -en el segundo- (Figura 10). 

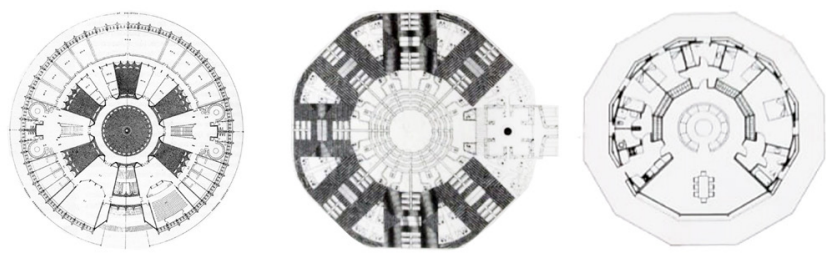

Figura 10: Plantas de la Corona de Espinas (1965), el proyecto de 200 viviendas Carmona y la vivienda unifamiliar en San Lorenzo de El Escorial (1978) [Fuente: Gazapo de Aguilera, D. y Lapayese Luque, C. (1997). Fernando Higueras, Arquitecturas. Madrid: Fundación Cultural COAM.

La innovación que supone este recurso no impide el uso de unas recurrencias estructurales previamente ensayadas que van desde el uso del hormigón, la inversión de las vigas y las galerías perimetrales hasta la introducción de jardineras interiores. Dentro de los aspectos constructivos diferenciales se encuentra la utilización de los materiales de la zona, una decisión derivada del empeño constante por adaptarse al entorno. Este objetivo, llevado al extremo, explica la elección del círculo - ejecutado de forma poligonal- como geometría para la planta. La visión total del paisaje y del interior que ofrece la radialidad de la casa va un paso más allá con respecto a las viviendas coetáneas en la asunción de las ideas de universalidad, originalidad y radicalidad, características fundacionales de la obra de Higueras y Miró. Vinculados a estos tres conceptos, la presencia del río y la fuente, la posición central de la chimenea y el tratamiento de la luz cenital, junto con la manipulación de la tierra hacen que, al recorrer la casa, se puedan identificar todos los elementos de la naturaleza conectados en una arquitectura esencial y primitiva que, sin embargo, no renuncia a una concepción radicalmente contemporánea del habitar.

El aislamiento físico de la vivienda ha permitido que su estado actual sea prácticamente el mismo que el original, a diferencia de las transformaciones integrales acometidas en Ciudad Aljarafe o el Club Social de Simón Verde. A pesar de ello, la desaparición de un valiosísimo patrimonio contemporáneo en contextos similares -desde la demolición en 1999 de la sede de los Laboratorios Jorba de Miguel Fisac a la Casa Guzmán de Alejandro de la Sota en 2017-, la ausencia de obras de conservación previstas o la presión inmobiliaria de la comarca del Aljarafe permiten asignarle un grado reseñable de vulnerabilidad. El proceso para su posible protección patrimonial comenzaría con su puesta en valor y difusión sobre la base de su carácter como pieza inalterada e inédita dentro del contexto contemporáneo andaluz, etapa sobre la que este artículo ha pretendido arrojar una primera luz.

\section{REFERENCIAS / REFERENCES}

(1) Gazapo de Aguilera, D. y Lapayese Luque, C. (1997). Fernando Higueras, Arquitecturas. Madrid: Fundación Cultural COAM.

(2) Humanes Bustamante, A. (1997). Fernando Higueras, arquitecto figurativo. En Fernando Higueras, Arquitecturas. Madrid: Fundación Cultural COAM.

(3) Higueras, F. y Botia, L. (1987). Fernando Higueras. Madrid: Xarait.

(4) García Torrente, U. y Reina Fernández, J. C. (1992). Guía de arquitectura de Sevilla y área metropolitana siglo xx. Sevilla: Colegio Oficial de Arquitectos de Andalucía Occidental, Demarcación de Sevilla, 158.

(5) Navarro-de-Pablos, J. (2017). La utopía de Ciudad Aljarafe: identidad vecinal frente a la ciudad dormitorio (pp. 67-81). En I Jornadas de Periferias Urbanas. Sevilla: Universidad de Sevilla. Escuela Técnica Superior de Arquitectura.

(6) García Martín, M. (2014). Transformaciones territoriales recientes en el Aljarafe sevillano: de la vocación rural a la integración metropolitana. Cuadernos Geográficos, 53(2): 25-53.

(7) Mayoral Campa, E., Navarro-de-Pablos, J., Pozo Bernal, M., Mayoral Moratilla, A. y Miró Miró, C. (2019). Aljarafe, más que un colegio: la arquitectura pedagógica de Fernando Higueras y Antonio Miró. Málaga: Recolectores Urbanos.

(8) García Millán, J. (2015). El Colegio Aljarafe: construir, vivir, enseñar, F. Higueras y A. Miró (pp. 47-60). En Espacios para la enseñanza. arquitecturas docentes de 6 arquitectos españoles de la 2. ${ }^{a}$ mitad del siglo xx. Madrid: Ediciones Asimétrica.

(9) J. Rufino Martín, comunicación personal, 19 de febrero de 2019.

(10) Mayoral Campa, E. y Pozo Bernal, M. (2015). Fernando Higueras, incursiones en el Sur: el Colegio Aljarafe. En International Conference Arquitectonics Network, 3-5 June 2015: Abstracts. Barcelona: GIRAS, Universitat Politècnica de Catalunya.

(11) Puntonet, J. A. (2016). La construcción del paisaje. Plan Parcial de Urbanización de Lanzarote. 1963. ZARCH: Journal of interdisciplinary studies in Architecture and Urbanism (7): 90-105.

(12) Higueras, F. y Miró, A. (1968). Casa Santoja, en Somosaguas, Madrid. Informes de la Construcción, 20 (199): 41-52.

(13) Botia, L. y Doval-Sánchez, G. (2019). Fernando Higueras: desde el origen. Madrid: Fundación ICO y Ministerio de Fomento.

(14) Higueras, F. y Miró, A. (1968). Memoria proyecto para vivienda unifamiliar en la Hacienda de las Teatinas en Palomares del Río (Sevilla). Fondo Fernando Higueras del Servicio Histórico del Colegio Oficial de Arquitectos de Madrid, ref. FH/Po27/Co10-2, consultado 19/10/2019.

(15) Del Blanco García, F. L. y García Ríos, I. (2018). Fernando Higueras y Félix Candela en un retorno a la geometría de paraguas invertidos. Análisis y reconstitución gráfica del aeropuerto de Murcia, 1983. EGA. Revista de Expresión Gráfica Arquitectónica, 23 (32), 232-243.

(16) Higueras, F. y Miró, A. (1970). Memoria Constructiva del Teatro principal de Burgos. Revista Nueva Forma (49): 21. 
(17) Puntonet, J. A. (2015). La construcción estructurante del espacio. Materialidad de la vivienda para Lucio Muñoz y Amalia Avia. Arquitecto Fernando Higueras. La relevancia del material. ZARCH Journal of interdisciplinary studies in Architecture and Urbanism (4): 72-85.

(18) García Ovies, A. (2015). El pensamiento creativo de Fernando Higueras (Tesis doctoral). Madrid: ETS Arquitectura (UPM). Consultado: http://oa.upm.es/34963. 